\title{
Implementación del Aprendizaje Móvil de la Plataforma Blackboard Mobile
}

. Ana Gabriela Bejarano* Olga Ligia Brenes Matarrita** Cinthya Valerio Álvarez***

\author{
*Productora Académica. Programa Aprendizaje en Línea. \\ Universidad Estatal a Distancia (UNED), Costa Rica; abejarano@uned.ac.cr \\ **Encargada de la Cátedra Tecnologías Informáticas para la Educación. \\ Universidad Estatal a Distancia (UNED), Costa Rica; obrenes@uned.ac.cr \\ ***Productora Académica. Programa Aprendizaje en Línea. \\ Universidad Estatal a Distancia (UNED), Costa Rica; cvalerio@uned.ac.cr
}

Recibido: 15 de noviembre de 2012

\section{RESUMEN}

Este artículo es un primer informe de investigación sobre una prueba piloto en el tema de aprendizaje móvil. El cual fue desarrollado por tres miembros del Programa de Informática Educativa y tres representantes del Programa Aprendizaje en Línea; que en conjunto conforma el equipo de investigación de Aprendizaje móvil de la Universidad Estatal a Distancia (UNED). Este estudio tuvo como objetivo conocer la percepción de los estudiantes y los profesores en relación con los beneficios, oportunidades y limitaciones en el uso de sistemas móviles (Mobile Learn) en la plataforma Blackboard Learnã de la Uned, los insumos permitirán valorar la posterior aplicación del sistema en la institución.

\section{PALABRAS CLAVE}

Aprendizaje móvil, educación a distancia, tecnologías de información y comunicación, sistema Blackboard Mobile

Aceptado: 20 de enero de 2013

\section{SUMMARY}

This article is the first report of a wider research on the topic of mobile learning, which is developing a team of three members of the Programa de Infomática Educativa and three represtatives Programa Aprendizaje en Línea, in collectively they make up an investigation team of Mobile Learning from the Universidad Estatal a Distancia (Uned). For this study, a pilot test was developed in order to study the perceptions of students and teachers regarding the benefits, opportunities and constraints, the use of mobile system (Mobile Learn) Blackboard Learn platform @ at Uned.

\section{KEY WORDS}

Mobile learning, distance education, information and communication technologies, Blackboard Mobile System

\section{INTRODUCCIÓN}

La cobertura telefónica móvil así como las características de los dispositivos móviles se conjugan para potenciar su uso en los modelos de educación a distancia, con el fin de proponer espacios donde se puede aprender en cualquier momento y lugar. Según la Rectoría de Telecomunicaciones (2012), la creciente incursión en el mercado nacional de dispositivos móviles ha abarcado al $82 \%$ de la pobla- 
ción costarricense. Aunado a esto, las características de conectividad, portabilidad e interactividad (UNESCO, s.f.) facilitan la integración de estos dispositivos en contextos educativos.

El uso de las Tecnologías de Información y Comunicación (TIC) cumple diferentes propósitos en contextos educativos, potenciando el proceso de enseñanza aprendizaje. Para los fines de este estudio es importante destacar que los sistemas de educación a distancia favorecen la comunicación entre los diferentes actores de dicho proceso. En este sentido, el modelo pedagógico de la UNED establece que: "La comunicación en diversas vías, a través de diferentes estrategias y de múltiples medios constituye, en este modelo pedagógico, el elemento central para el logro del aprendizaje, la comprensión y el desarrollo del discurso como manifestación del aprendizaje" (UNED, 2004, p. 20).

Partiendo de este principio y con el fin de facilitar la integración de las TIC, los modelos de educación a distancia deberán aprovechar los beneficios de las tecnologías que ya utilizan los estudiantes, y que pueden ser consideradas en los procesos de aprendizaje. Por ello, es indispensable realizar investigaciones sobre las tecnologías emergentes con el fin de valorar su pertinencia pedagógica y efecto en la reducción de la distancia relacional entre los actores del proceso educativo.

Por estas razones, se consideró importante realizar una prueba piloto para valorar los beneficios, oportunidades y limitaciones del uso del sistema móvil (Mobile Learn) de la plataforma BlackboardLearn@ en la UNED. Los principales resultados se muestran en este artículo con el propósito de aclarar y exponer las conclusiones más relevantes.

\section{¿Qué es el sistema Blackboard Mobile@?}

BlackboardLearningSystem $\odot$ es una plataforma informática para procesos de enseñan- za y aprendizaje en línea. Contiene una serie de herramientas con las cuales se planifican y desarrollan diversas estrategias de formación, comunicación, mediación y evaluación de cursos en línea; tales como: enlaces web, foros, correos, exámenes, entre otros.

Dentro de las opciones que ofrece BlackboardLearningSystem $\odot$, está el sistema Blackboard Mobile@, el cual permite acceder a esta plataforma desde dispositivos móviles a través de una aplicación que modifica la visualización de la interfaz web, adaptándola a las características y tamaño del dispositivo. Al sistema de BlackboardLearn, se le integra la aplicación de Blackboard Mobile, la cual utiliza un servidor y base de datos independiente para el manejo de este sistema y permite la posibilidad de ser visualizado desde los sistemas operativos iOs, Android y Blackberry.

El Cuadro 1 muestra las posibilidades que brinda el sistema Mobile Learn de Blackboard, tanto para estudiantes como para profesores, tomando en cuenta que algunas de estas actividades pueden ser realizadas por los profesores solamente, dado el rol que cumplen en el curso. Por ejemplo, los estudiantes no pueden enviar anuncios a los demás compañeros o publicar materiales de estudio en formato de video o audio, porque esta es una función del profesor, a menos que se les solicite como parte de una tarea.

\section{- Blackboard Mobile}

El manejo y visualización de la interfaz de la plataforma LMS varía si se observa desde un dispositivo móvil o desde un computador personal. A continuación se describen los principales elementos de la interfaz de Blackboard Mobile.

\section{Interfaz de entrada}

Los usuarios de la plataforma BlackboardLearn( $\odot$ acceden a su curso a través del App, el cual está disponible para los sistemas 
Cuadro 1. Posibilidades del sistema Mobile Learn de Blackboard

\begin{tabular}{|l|c|c|}
\hline \multicolumn{1}{|c|}{ Criterios } & Estudiantes & Profesores \\
\hline Recibir notificaciones cuando las actividades de curso estén por vencer. & $\checkmark$ & \\
\hline Enviar anuncios. & & $\checkmark$ \\
\hline Revisar anuncios. & $\checkmark$ & $\checkmark$ \\
\hline Publicar archivos de audio, video y texto, en diferentes formatos. & & $\checkmark$ \\
\hline Acceder a archivos de audio, video y texto, en diferentes formatos. & $\checkmark$ & $\checkmark$ \\
\hline Visualizar y participar en foros de discusión. & $\checkmark$ & $\checkmark$ \\
\hline Comentar en blogs y diarios. & $\checkmark$ & $\checkmark$ \\
\hline Ver calificaciones. & $\checkmark$ & \\
\hline Filtrary marcar la lista de cursos favoritos. & $\checkmark$ & $\checkmark$ \\
\hline
\end{tabular}

Fuente: Blackboard, 2012.

Android, iOS y Blackberry, lo que le permite observar sus contenidos adaptados para las pantallas de los diferentes dispositivos móviles. Lo anterior facilita que se descargue y adjunten diferentes archivos.

La aplicación posee un espacio para incluir el nombre de la universidad, pero dado que la UNED no cuenta con el licenciamiento de Blackboard Mobile, la compañía asignó el código 1jdvzz para poder acceder a la base de datos de Blackboard y posteriormente digitar el nombre de usuario y contraseña (véase Figura 1 y 2 ).

\section{Descripción del APP de Blackboard}

Desde esta aplicación el estudiante puede acceder a la lista de los cursos que tiene matriculados e ingresar a las diferentes herramientas que se emplearon como actividades en el curso tales como: avisos, foros, blogs, tareas y archivos adjuntos. Además, la aplicación móvil permite, tanto a estudiantes como a profesores, adjuntar y descargar videos, documentos e imágenes en diversos formatos.

\section{METODOLOGÍA}

El estudio sobre la Implementación del Aprendizaje Móvil de la Plataforma Blackboard Mobile consistió en el uso de la herramienta de aprendizaje móvil de la plataforma con un grupo de estudiantes de la UNED, inscritos en cursos en línea durante el primer cuatrimestre del año 2012, específicamente durante un mes (12 de febrero a 12 de marzo). Posteriormente, se aplicó una encuesta a toda esa población estudiantil y a los tutores para conocer la percepción que ellos presentaban con respecto al uso de la plataforma para el aprendizaje móvil. De la totalidad de la población, que estuvo conformada por 3256 personas, se obtuvo respuesta de 304, por lo que los resultados expresan las opiniones únicamente de esas personas que contestaron el cuestionario.

El estudio se realizó de acuerdo con los siguientes procedimientos:

1. Consecución de autorizaciones institucionales y del proveedor para utilizar la plataforma de aprendizaje móvil por un periodo de un mes. 


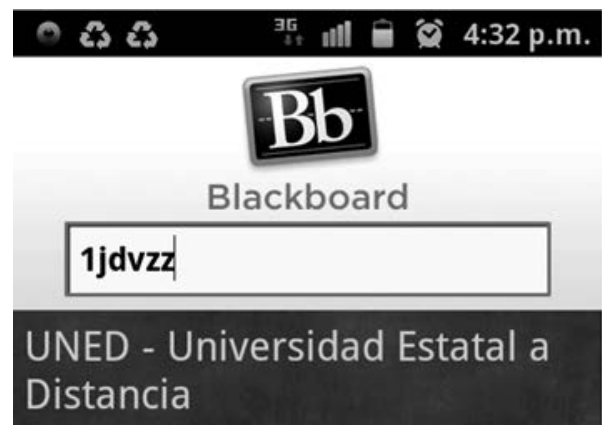

Figura 1. Código para acceder a Blackboard Mobile de la UNED.

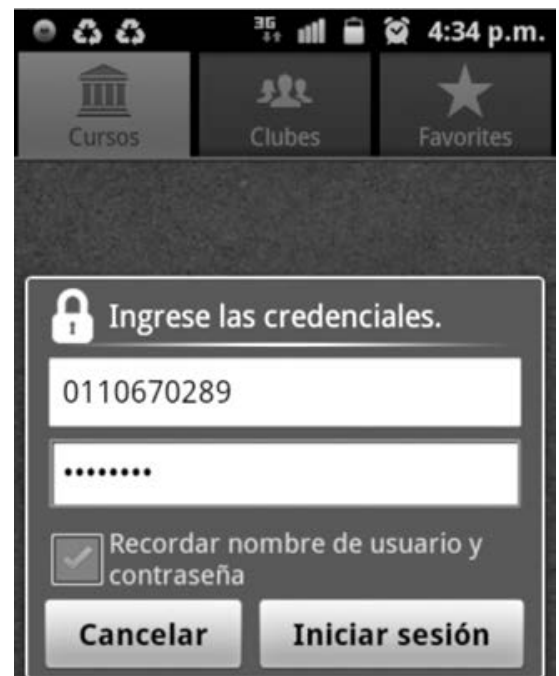

Figura 2. Ingreso del usuario y contraeña del sistema.
2. Integración de la plataforma para el aprendizaje móvil al servidor institucional en el que se hospeda BlackboardLearn.

3. Diseño de manuales para acceder a la aplicación Mobile desde los sistemas operativos iOS y Android.

4. Divulgación, mediante correo electrónico personal y mediante anuncio página web y en el sitio del PAL en Facebook, de la disponibilidad de uso de la plataforma de aprendizaje móvil.

5. Diseño y validación del cuestionario que se utilizaría para conocer la percepción de los estudiantes y profesores. Una vez validada se dispuso en un software para la realización de encuestas en línea (Checkbox).

6. Solicitud, a la población inscrita en los cursos en línea y a los docentes, para que llenaran la encuesta en línea.

7. Migración de los datos recuperados mediante la encuesta a una base de datos en Excel para la realización de los respectivos análisis estadísticos.
La prueba piloto se desarrolló de la siguiente manera:

1. El Programa de Aprendizaje en Línea (PAL) solicitó a Blackboard Inc. los permisos para la integración del servicio móvil al servidor de BlackboardLearn de la UNED, por un plazo de un mes.

2. La Vicerrectoría Académica firmó un contrato con Blackboard Inc. para realizar una prueba piloto con Blackboard Mobile.

3. Blackboard Inc. integró la plataforma de Blackboard Mobile al servidor de BlackboardLearn en la UNED.

4. EI PAL produjo los manuales para acceder a la aplicación móvil, desde los sistemas operativos iOS y Android. Estos manuales se publicaron en la página web del PAL.

5. Los administradores de la plataforma enviaron un correo electrónico a todos los estudiantes matriculados, en la plataforma BlackboardLearn (c) en el 
primer cuatrimestre 2012, informándoles sobre el inicio de la prueba piloto y la ubicación de los manuales para instalar la aplicación. Además, la divulgación se realizó a través de la página del Programa de Aprendizaje en Línea, Facebook y de la plataforma BlackboardLearn $\odot$.

6. La comisión de investigación del proyecto, diseñó y validó una encuesta de opinión autoadministrada dirigida a estudiantes y profesores inscritos en los cursos de BlackboardLearn@.

7. Se empleó la herramienta Checkbox, para que la encuesta estuviera accesible en línea.

8. Los administradores de la plataforma enviaron un mensaje a todos los usuarios de Blackboard, notificando la dirección electrónica de la encuesta. La divulgación se realizó a través de la página del Programa de Aprendizaje en Línea, Facebook y de la plataforma BlackboardLearn@.

9. Una vez finalizado el periodo para completar la encuesta, los resultados se exportaron a una hoja de cálculo de Excel@.

10. Con la información recopilada, se realizó un análisis estadístico.

\section{- Selección de la muestra}

Para este pilotaje se consideró la población total de estudiantes y profesores del período académico comprendido entre los meses de febrero y marzo del $2012(N=3256)$ en los 254 cursos que se ofertan en la plataforma BlackboardLearn $\odot$. La muestra estuvo constituida por los estudiantes y profesores que de manera voluntaria respondieron el cuestionario autoadministrado $(n=304)$.

\section{- Descripción del instrumento}

El instrumento utilizado para la recolección de información consistió en un cuestionario diseñado para ser autoadministrado, constituido por catorce ítems, de los cuales diez fueron de selección única y cuatro preguntas abiertas. Los ítems se orientaron a recolectar información referente $a$ :

1. Datos demográficos: sexo y edad.

2. Tenencia de dispositivos móviles: marca, modelo y sistema operativo.

3. Uso y percepción: sobre la experiencia de aprendizaje al hacer uso de Mobile Learning.

El cuestionario fue validado por especialistas en educación y tecnólogos educativos, ambos grupos tienen conocimiento en el uso de la plataforma BlackboardLearn@.

\section{RESULTADOS Y ANÁLISIS}

\section{Características de los encuestados}

Se registraron respuestas de 304 encuestados, de los cuales un $85,1 \%$ son estudiantes, un $11,6 \%$ son tutores y un $3,3 \%$ indica como "otra" la función que ejerce en la universidad. El rango de edad con mayor porcentaje se ubica entre los 28 y 37 años $(39,1 \%)$, en segundo lugar se ubican los sujetos con edades entre 18 y 27 años (24,7\%). El $44,7 \%$ de los encuestados pertenece al género masculino y el $55,3 \%$ al género femenino.

\section{- Características de los dispositivos móviles}

Con respecto a la marca del dispositivo que emplean los encuestados para acceder al 
aprendizaje móvil en la plataforma Blackboard, se observan los resultados en el Cuadro 2.

A partir de la información obtenida en el Cuadro 2, el grupo de investigación determinó el sistema operativo del dispositivo utilizado por los encuestados.

En la Figura 3 se observa el porcentaje de los sistemas operativos más utilizados por los usuarios.

A partir de estos datos se puede concluir que un $77 \%$ de los encuestados utiliza teléfonos tipo smartphone, lo cual significa que podían tener acceso a Internet y contar con la posibilidad de descargar aplicaciones para sus dispositivos.

Al realizar el análisis de modelos de los dispositivos móviles con los que accedían los encuestados a la aplicación móvil de Blackboard, se evidenció que un $83 \%$ accede desde smartphones, $9 \%$ desde tabletas, $7 \%$ desde celulares y un $1 \%$ lo hace desde una Palm. Se comprobó que la mayoría de los encuestados ingresa a la plataforma desde sus teléfonos móviles, por lo tanto, la producción de materiales y diseños de interfaces debe enfocarse prioritariamente, a estos dispositivos.

\section{Acceso al sistema de aprendizaje móvil de la plataforma Blackboard}

El 75\% de los participantes no presentó problemas para acceder al servicio de aprendizaje móvil, mientras que el $25 \%$ manifestó lo contrario. Dentro de las limitaciones, los encuestados manifestaron:

» no tener acceso a Internet

» dificultad para encontrar el App de Blackboard

» el código de ingreso de la universidad no es fácil de recordar

» problemas para acceder al App por el tipo de dispositivo que poseía.

Cuadro 2. Marca de los dispositivos móviles utilizados por los encuestados

\begin{tabular}{|l|c|c|}
\hline \multicolumn{1}{|c|}{ Marca del dispositivo } & Porcentaje & Valor absoluto \\
\hline Apple - iPhone & 26,8 & 79 \\
\hline BlackBerry & 1,4 & 4 \\
\hline HTC & 1 & 3 \\
\hline Huawei & 2,7 & 8 \\
\hline Lenovo & 0,7 & 2 \\
\hline LG & 0,7 & 2 \\
\hline Motorola & 1,4 & 4 \\
\hline Nokia & 14,2 & 42 \\
\hline Samsung & 12,9 & 38 \\
\hline Sony Ericsson & 8,1 & 24 \\
\hline ZTE & 0,3 & 1 \\
\hline Otro & 29,8 & 88 \\
\hline
\end{tabular}

Fuente: Encuesta a estudiantes plan piloto de aprendizaje móvil de Blackboard, 2012. 


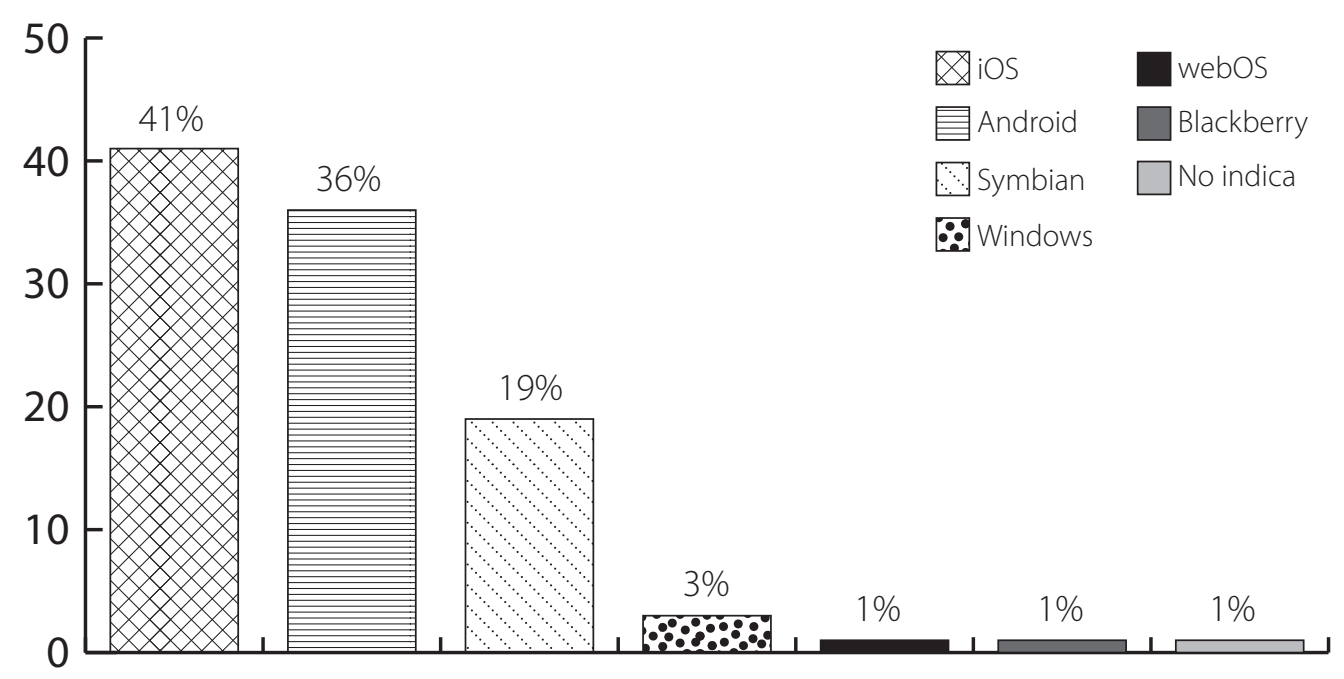

Figura 3.Sistemas operativos según los modelos de los dispositivos de los encuestados.

En el Cuadro 3 se detallan los problemas que enfrentaron los encuestados para acceder al servicio.

Se observa que, de la totalidad de criterios, la mayoría corresponde a factores externos a la universidad $(80,26 \%)$, lo que significa que el éxito de un proyecto para la incorporación de tecnologías móviles estará sujeto también a las posibilidades de los estudiantes de contar con conectividad o acceso a Internet y dispositivos de tercera generación. El 11,84\% no especifica las limitaciones. Un porcentaje menor (7,9\%), corresponde a limitaciones que la universidad puede subsanar.

\section{- Acceso a actividades y materiales del curso por medio del sistema de aprendizaje móvil}

El $72 \%$ de los encuestados manifestaron tener acceso a los materiales y actividades del curso desde su dispositivo, mientras que un $28 \%$ respondió de forma negativa. El porcentaje anterior que no pudo acceder a los materiales y actividades, indicó diferentes causas:

» problemas de conexión a internet.

» incompatibilidad del sistema operativo.

» desconocimiento y errores en la digitación del código de ingreso a la UNED, entre otros.

Los encuestados destacan que el acceso a los materiales y actividades del curso desde el dispositivo móvil es una de las razones más significativas para su uso.

\section{- Percepción de la utilidad del sistema Blackboard Mobile}

Puede observarse, en la figura 4, que la respuesta con mayor incidencia coincide con la característica principal del aprendizaje móvil, la cual es que los estudiantes puedan revisar los materiales del curso en cualquier momento y lugar. 
Cuadro 3. Dificultades para acceder al sistema de aprendizaje móvil

\begin{tabular}{|l|c|c|}
\hline \multicolumn{1}{|c|}{ Dificultad } & Porcentaje & Valor absoluto \\
\hline No tengo acceso a Internet. & 23,69 & 18 \\
\hline No encontró con facilidad el APP o aplicación. & 21,05 & 16 \\
\hline La descarga del APP fue difícil. & 21,05 & 16 \\
\hline Mi dispositivo no tiene esa posibilidad. & 14,47 & 11 \\
\hline $\begin{array}{l}\text { Los manuales ofrecidos en la página de aprendizaje en } \\
\text { línea para la instalación del APP no fueron entendibles. }\end{array}$ & 7,90 & 6 \\
\hline Otra. & 11,84 & 9 \\
\hline
\end{tabular}

Fuente: Encuesta a estudiantes plan piloto de aprendizaje móvil de Blackboard, 2012.

Revisión del curso (materiales de apoyo al estudio e ingreso a actividades) en cualquier momento y lugar, sin depender de la computadora

: El sistema es sencillo y de fácil uso
Agilidad en la comunicación

Ahorro de tiempo

Otros

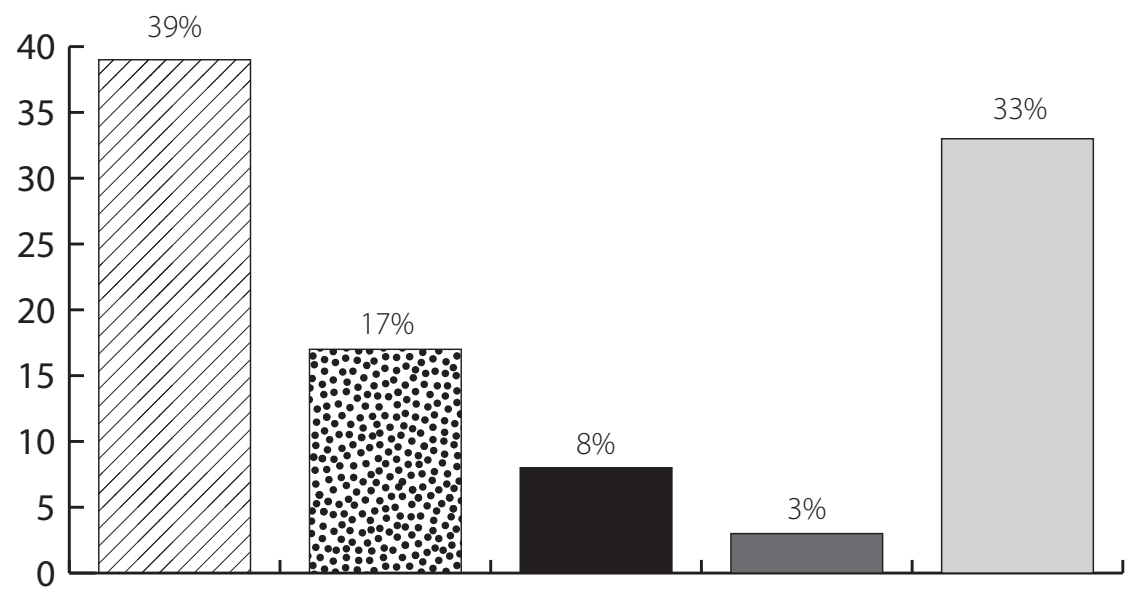

Figura 4.Percepción de la utilidad del aprendizaje móvil.

\section{CONCLUSIONES} Y RECOMENDACIONES

La mayor utilidad que los encuestados destacaron durante la prueba piloto desarrollada se basó en la característica de ubicuidad que experimentaron al accesar a los cursos virtuales desde sus dispositivos móviles, es decir, el poder tener la disponibilidad de acceso a los recursos y actividades de aprendizaje en todo momento y lugar.

Los sistemas operativos que más utilizan los encuestados son iOS y Android, los cuales se encuentran en sus smartphones. Se pudo evidenciar que el uso de tabletas aún no se ha 
generalizado entre la población encuestada. Se recomienda que la planificación para el desarrollo de aplicaciones para dispositivos móviles en una primera etapa, deba ser compatible con los sistemas operativos iOS y Android, enfocada de manera específica a smartphones, para aprovechar los dispositivos móviles que ya se están utilizando.

La mayor parte de los encuestados manifestó no tener problemas para acceder a los materiales y actividades de los cursos desde sus dispositivos móviles. De las personas que reportaron problemas, estos son ajenos al propio sistema de la plataforma Blackboard Mobile, ya que se mencionaron: el acceso a Internet, desconocimiento de cómo funcionan las aplicaciones para dispositivos móviles e incompatibilidad con los sistemas operativos. En este sentido se recomienda que la institución planifique estrategias tendientes a facilitar el acceso inalámbrico desde sus diferentes sedes, de manera que se solvente uno de los problemas manifestados.

Adicionalmente, con el fin de disminuir la brecha de acceso y las posibilidades de tenencia de dispositivos inteligentes por parte de estudiantes y tutores, se recomienda establecer convenios y alianzas estratégicas con proveedores de servicios de telefonía celular que garanticen el ofrecimiento de condiciones favorables para poder adquirir este tipo de dispositivos y sus servicios.

Dado que algunos estudiantes manifestaron tener problemas para descargar el APP de Blackboard, se recomienda evaluar los manuales realizados con este fin y analizar las posibilidades de crear materiales redundantes en otros formatos.
Las limitaciones reportadas por los estudiantes en el uso de aprendizaje móvil dejan en evidencia la necesidad de una alfabetización tecnológica obligatoria, tanto para docentes como para estudiantes, misma que debe programarse al ingresar a la institución por primera vez.

El grado de satisfacción de los usuarios con respecto a la utilidad de la aplicación Blackboard Mobile desde el dispositivo móvil fue valorado, en forma general, de manera positiva. Debido a estos resultados, se recomienda continuar con el desarrollo de investigaciones que cubran estas temáticas y avanzar hacia el diseño de propuestas sobre la implementación pedagógica de tecnologías móviles en el contexto educativo de la institución.

\section{REFERENCIAS}

Blackboard (2012). What Can You Do With Blackboard Mobile Learn? Recuperado de: http:// www.blackboard.com/resources/mobile/mobile_learn_splash/desktop/portal-nonsprint html\#android.

Rectoría de Telecomunicaciones (2012). Telefonía móvil e Internet móvil en Costa Rica. Recuperado de http://www.telecom.go.cr/index.php/ publicaciones2/publicaciones/cat_view/52publicaciones/74-boletines

UNESCO (s.f.). El aprendizaje móvil. Recuperado de: http://www.unesco.org/new/es/unesco/ themes/icts/m4ed/

Universidad Estatal a Distancia (2004). Modelo Pedagógico. EUNED. 
